\title{
Características de carcaça de ovelhas de descarte das raças Ideal e Texel terminadas em dois sistemas de alimentação
}

\section{Luis Fernando Vilani de Pelegrini ${ }^{1}$, Cleber Cassol Pires ${ }^{2}$, Diego Barcelos Galvani ${ }^{3}$, Anderson Michel Soares Bolzan ${ }^{4}$, Guilherme Coradini Fontoura da Silva ${ }^{5}$}

\footnotetext{
${ }^{1}$ Departamento de Medicina Veterinária Preventiva, UFSM.

2 Departamento de Zootecnia, UFSM. Pesquisador do CNPq.

3 Programa de Pós-graduação em Ciência Animal e Pastagens, ESALQ/USP

${ }^{4}$ Curso de Zootecnia, UFSM.

${ }^{5}$ Curso de Medicina Veterinária, UFSM
}

RESUMO - Avaliaram-se as características de carcaça de ovelhas de descarte das raças Ideal e Texel terminadas em dois sistemas de alimentação. Utilizaram-se 20 ovelhas (10 da raça Ideal e 10 da raça Texel) aleatoriamente distribuídas de acordo com o grupo genético em dois manejos alimentares (confinamento e pastagem cultivada). Os abates foram realizados quando os animais atingiram 3,5 pontos de condição corporal. Os pesos de carcaça quente (PCQ) e de carcaça fria (PCF) e os rendimentos de carcaça quente (RCQ) e de carcaça fria (RCF) das ovelhas Texel foram maiores que os das ovelhas Ideal: 27,85 e $19,04 \mathrm{~kg} ; 27,08$ e $18,43 \mathrm{~kg} ; 47,25$ e 45,20\% e 45,95 e 43,72\%, respectivamente. O peso dos cortes perna, paleta, pescoço e costela e os pesos de músculos, ossos e gordura da perna dos animais da raça Texel foram superiores aos obtidos na raça Ideal. Quando avaliadas em porcentagem do peso corporal, no entanto, essas características não diferiram entre as duas raças. O manejo alimentar não influenciou as características de carcaça de ovelhas de descarte. Ovelhas Texel apresentam cortes maiores que os de ovelhas Ideal.

Palavras-chave: genótipo, manejo alimentar, ovelha de descarte, ovinos, rendimento

\section{Carcass traits of culling ewes from Texel and Polwarth breeds submitted to two feeding systems}

\begin{abstract}
Carcass traits of culling ewes from Texel and Polwarth breeds submitted to two feeding systems were evaluated. Twenty ewes (10 from Polwarth breed and 10 from Texel breed) were randomly distributed according to its genetic group, in two feeding systems (feedlot and cultivated pasture). The slaughter was carried out when the animals reached body condition score of 3.5 points. Hot carcass (HCW) and cold carcass (CCW) weights and hot carcass (HCD) and cold carcass (CCD) dressing of Texel ewes were higher than those of Polwarth ewes: 27.85 and $19.04 \mathrm{~kg}, 27.08$ and $18.43 \mathrm{~kg}, 47.25$ and 45.20 and 45.95 and $43.72 \%$, respectively. The absolute weights of cuts for leg, shoulder, neck and rib, and absolute weights for muscles, bones and leg fat of ewes from Texel breed were higher than those obtained by Polwarth breed. When evaluated as relative weights, however, these characteristics did not differ between the two breeds. The feeding system did not constitute a change factor for carcass traits of culling ewes. Texel ewes breed showed cuts with higher weights than those obtained by Polwarth ewes breed.
\end{abstract}

Key Words: culling ewe, dressing percentage, feeding management, genotype, sheep

\section{Introdução}

O significativo aumento na demanda de carne ovina nos últimos anos, sobretudo nos grandes centros urbanos, torna evidente a necessidade de produzir animais que atendam às exigências desses mercados, que têm demonstrado preferência por animais jovens, com aproximadamente 150 dias de idade e de 12 a $14 \mathrm{~kg}$ de peso de carcaça (Siqueira, 1999). O descarte de ovelhas do rebanho de cria, no entanto, é uma prática rotineira em propriedades com ciclo completo de produção, onde a comercialização desses animais é dificultada pela baixa aceitabilidade da sua carne pelo mercado consumidor.

A aplicação de tecnologias para agregar valor à carne desses animais pode, além de proporcionar maior 
facilidade de comercialização, aumentar a lucratividade dos sistemas de produção. Nesse contexto, a fabricação de embutidos fermentados e/ou de cortes defumados pode ser uma boa alternativa, mas exige o prévio conhecimento das características de carcaça dos animais.

O rendimento de carcaça geralmente é o índice mais importante para estimar o valor comercial da carcaça (Sainz, 1996). De acordo com Huidobro \& Cañeque (1993), a proporção de cada componente, do mesmo modo, é essencial na avaliação de carcaça. A perna é o corte de maior valor comercial, uma vez que é composta, em grande parte, por tecidos comestíveis.

A gordura é o tecido de maior variabilidade no animal, tanto em quantidade como em distribuição. Ferreira et al. (2001) ressaltaram que o teor de gordura da carcaça afeta diretamente sua aceitabilidade, principalmente em virtude da preocupação dos consumidores com os efeitos nocivos do consumo excessivo de alimentos com alta densidade calórica na saúde humana. Raça e sistema alimentar (Neres et al., 2001), assim como idade e sexo do animal (Rosa et al., 2002), são os fatores que mais afetam a composição física da carcaça de ovinos.

Este trabalho foi realizado para avaliar a influência do sistema de alimentação na fase de terminação sobre as características de carcaça de ovelhas de descarte de dois grupos genéticos.

\section{Material e Métodos}

O experimento foi conduzido no Laboratório de Ovinocultura do Departamento de Zootecnia da Universidade Federal de Santa Maria, em Santa Maria, Rio Grande do Sul, nos meses de julho de 2005 a maio de 2006. A região, fisiograficamente denominada Depressão Central, está situada a $29^{\circ} 42^{\prime} \mathrm{S}$ de latitude, $53^{\circ} 48^{\prime} \mathrm{W}$ de longitude e $95 \mathrm{~m}$ de altitude. O clima, segundo a classificação de Köppen, é o do tipo Cfa, subtropical úmido (Moreno, 1961).

Foram utilizadas 20 ovelhas ( 10 da raça Ideal e 10 da raça Texel) com aproximadamente 7 anos de idade e condição corporal 2 em escala de 1 a 5 ( 1 - muito magra e 5 - muito gorda; Russel et al., 1969). As ovelhas foram aleatoriamente distribuídas, de acordo com o grupo genético, em dois sistemas de alimentação: confinamento e pastagem cultivada.

A pastagem foi cultivada com preparo mínimo do solo, utilizando-se mistura de aveia-preta (Avena strigosa Schreb.) e azevém-anual (Lolium multiflorum Lam.) em densidades de sementes de 70 e $25 \mathrm{~kg} / \mathrm{ha}$, respectivamente. A adubação de base foi realizada com adubo NPK 5-20-20
(200 kg/ha) e nitrogênio (90kg/ha), na forma de uréia, em três aplicações. A área total da pastagem foi subdividida em dois piquetes de $0,40 \mathrm{ha}$, onde foram alocadas, em cada um dos piquetes, cinco ovelhas-teste e um número variável de ovelhas reguladoras (Mott \& Lucas, 1952), visando manter oferta de forragem de aproximadamente $10 \mathrm{~kg}$ de MS/100 kg PV/dia. A massa de forragem (MF) foi determinada no início do experimento e, posteriormente, a cada 21 dias, pela técnica de dupla amostragem (Wilm et al., 1944). As amostras coletadas foram separadas manualmente, obtendo-se as frações folha, haste e material morto. $\mathrm{O}$ valor médio obtido para massa de folhas verdes durante o período experimental foi de $1.043 \mathrm{~kg}$ de MS/ha.

A dieta fornecida aos animais confinados, calculada de acordo com os requerimentos especificados pelo NRC (1985), foi composta de 59,5\% de silagem de sorgo (Sorghum bicolor (L.) Moench), 25,5\% de grão de milho (Zea mays L.) triturado, 13,8\% de farelo de soja (Glycine max L.), 0,6\% de sal comum, 0,54\% de calcário calcítico e 0,10\% de fosfato bicálcico, totalizando 14,3\% de proteína bruta, 37,2\% de fibra em detergente neutro e 2,51 Mcal de EM/kg de MS. Os animais foram alimentados à vontade duas vezes ao dia, às 8 e $16 \mathrm{~h}$.

Os abates foram realizados após jejum de sólidos por 14 horas, assim que cada grupo de animais atingiu o escore corporal 3,5, registrando-se o peso vivo ao abate (PA). Após esfola e evisceração, as carcaças foram imediatamente pesadas para obtenção do peso de carcaça quente (PCQ) e do rendimento de carcaça quente (RCQ) e armazenadas em câmara frigorífica a $2^{\circ} \mathrm{C}$ durante 24 horas. Depois de resfriadas, as carcaças foram novamente pesadas para obtenção do peso de carcaça fria (PCF), do rendimento de carcaça fria (RCF) e do índice de quebra ao resfriamento (IQ).

Posteriormente, as carcaças foram seccionadas longitudinalmente e, na metade esquerda, foram mensurados o comprimento de carcaça (distância entre o bordo anterior da sínfise isquiopubiana e o bordo anterior da primeira costela no seu ponto médio), o comprimento de perna (distância entre o bordo anterior da sínfise isquiopubiana e a porção média dos ossos do tarso), a largura de perna (distância entre os bordos interno e externo da parte superior da perna em sua parte mais larga), a profundidade de perna (distância entre os bordos proximal e distal da parte superior da perna em sua parte mais larga) e a profundidade de peito (distância máxima entre o dorso e o esterno). As avaliações foram realizadas segundo metodologias descritas por Osório et al. (1998).

A área de olho-de-lombo foi obtida pela exposição do músculo Longissimus dorsi após um corte transversal na 
carcaça, entre a $12^{\underline{a}}$ e $13^{\underline{a}}$ costela, traçando o seu contorno em papel-vegetal (Müller, 1980). Para determinação e registro da área, utilizou-se o programa SITER 3.1, modelo A2, descrito por Giotto (2001).

Em seguida, a meia-carcaça direita foi subdividida em quatro regiões anatômicas: perna, paleta, costela e pescoço (Osório et al., 1998). Depois de pesadas as partes, a perna foi dissecada em ossos, músculos e gordura e cada componente foi pesado separadamente e expresso em valores absolutos ( $\mathrm{kg}$ ) e relativos (\% do peso da perna).

O delineamento experimental utilizado foi o inteiramente casualizado em arranjo fatorial $2 \times 2$, composto de duas raças e dois sistemas de alimentação. Os dados foram submetidos à análise de variância, cujo modelo incluiu os efeitos de raça, sistema alimentar e interação raça $\times$ sistema alimentar e o erro experimental, e ao teste $\mathrm{F}$ a $5 \%$ de probabilidade. Quando observadas interações significativas, as médias foram comparadas pelo teste de Tukey, também a 5\% de probabilidade. As análises foram realizadas com auxílio do pacote estatístico SAS (2004).

\section{Resultados e Discussão}

Os animais da raça Texel apresentaram pesos ao início e ao final do período experimental, em média, 39,8\% superiores aos obtidos nos animais da raça Ideal (Tabela 1), o que está relacionado à aptidão produtiva dessas raças. Do mesmo modo, o ganho médio diário (GMD) das ovelhas Texel foi significativamente maior $(\mathrm{P}<0,01)$ que o das ovelhas Ideal, contudo, esse maior ganho de peso não refletiu no número de dias necessários para que os animais atingissem a condição corporal predeterminada para abate $(3,5)$. De acordo com o ARC (1980), maiores taxas de ganho resultam em maior deposição de gordura corporal. Assim, esperava- se que os animais da raça Texel atingissem a condição de abate em menor número de dias. Entretanto, segundo o ARC (1980), animais de raças destinadas à produção de lã, como a Ideal, apresentam, em mesma taxa de ganho, maior deposição de gordura corporal em comparação às produtoras de carne, o que justifica a semelhança no tempo gasto pelas ovelhas Ideal e Texel utilizadas nesta pesquisa para atingir a condição corporal de abate, apesar da menor taxa de ganho das ovelhas Ideal.

As ovelhas terminadas em confinamento atingiram em menor tempo a condição de abate $(\mathrm{P}<0,05)$, o que provavelmente está relacionado aos ganhos médios diários, que, entretanto, não diferiram $(\mathrm{P}>0,05)$ entre os sistemas de terminação. A taxa média de ganho observada neste estudo $(0,165 \mathrm{~kg} / \mathrm{dia})$ foi superior à relatada por Frescura et al. (2005) (0,066 kg/dia), contudo, esses autores estudaram ovelhas em fase de lactação.

Não foi observada interação raça $\times$ sistema de alimentação $(\mathrm{P}>0,05)$ para os pesos de carcaça quente (PCQ) e de carcaça fria (PCF), os rendimentos de carcaça quente (RCQ) e de carcaça fria (RCF) e o índice de quebra ao resfriamento (IQ) (Tabela 2). O grupo genético, no entanto, teve efeito significativo sobre essas variáveis, exceto sobre o índice de quebra ao resfriamento.

Os maiores pesos de carcaça quente e fria das ovelhas Texel estão relacionados ao seu maior peso de abate. Em valores percentuais, as ovelhas Texel foram 39,82\% (PA), 46,27\% (PCQ), 46,93\% (PCF), 4,53\% (RCQ) e 5,10\% (RCF) superiores às da raça Ideal. Os valores obtidos para essas características de carcaça foram pouco inferiores aos encontrados por Osório et al. (1996), que verificaram superioridade de 42,80\% (PA), 51,96\% (PCQ), 52,80\% (PCF), 6,33\% (RCQ) e 6,90\% (RCF) de cordeiros da raça Texel em relação aos da raça Ideal.

Tabela 1 - Desempenho de ovelhas Ideal e Texel terminadas em dois sistemas de alimentação

\begin{tabular}{|c|c|c|c|c|}
\hline & Peso inicial (kg) & Peso ao abate (kg) & Dias para o abate & Ganho médio diário (kg/dia) \\
\hline Ideal & 35,7 & 42,1 & 47,6 & 0,136 \\
\hline \multicolumn{5}{|c|}{ Sistema alimentar } \\
\hline Confinamento & 41,2 & 49,5 & 46,0 & 0,183 \\
\hline Pastagem & 44,3 & 51,6 & 49,0 & 0,147 \\
\hline $\mathrm{P}^{\mathrm{a}}$ & ns & ns & ns & ns \\
\hline Média & 42,7 & 50,5 & 47,5 & 0,165 \\
\hline $\mathrm{CV}, \%$ & 10,22 & 9,01 & 5,88 & 28,84 \\
\hline
\end{tabular}

a Probabilidade dos efeitos de raça, sistema alimentar e da interação raça $x$ sistema alimentar, em que ns = não-significativo, *P<0,05 ** $\mathrm{P}<0,01$ e *** $\mathrm{P}<0,001$ $\mathrm{CV}=$ coeficiente de variação. 
Tabela 2 - Pesos de carcaça quente (PCQ) e fria (PCF), rendimentos de carcaça quente (RCQ) e fria (RCF) e índice de quebra ao resfriamento (IQ) de ovelhas Ideal e Texel terminadas em dois sistemas de alimentação

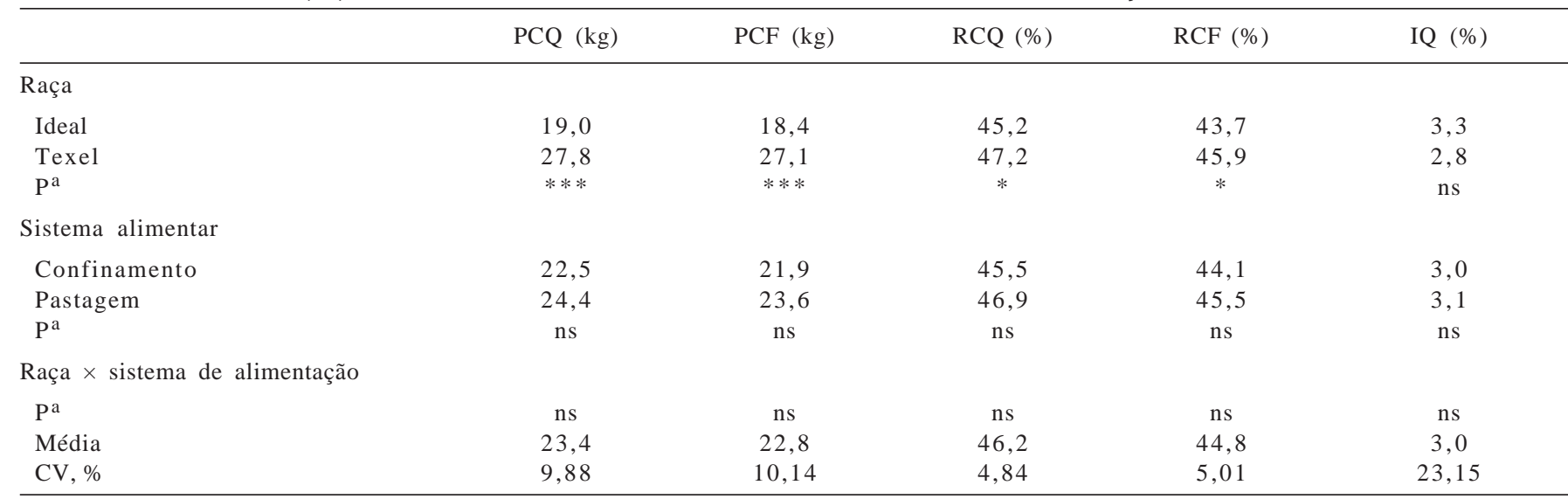

a Probabilidade dos efeitos de raça, sistema alimentar e da interação raça $\times$ sistema de alimentação, em que ns $=$ não significativo, * $P<0,05$ e ***P<0,001 $\mathrm{CV}=$ coeficiente de variação.

Apenas os grupos genéticos tiveram efeito significativo sobre as medidas da carcaça (Tabela 3). As ovelhas Texel apresentaram maior área de olho-de-lombo, o que, de acordo com Macedo et al. (2000), é um indicativo da maior quantidade de músculo na carcaça desses animais e está relacionada à maior aptidão desta raça para produção de carne (Furusho-Garcia et al., 2000; Jones et al., 2002).

O genótipo é o fator que mais influencia as características de carcaça de ovelhas de descarte (Tabelas 2 e 3), no entanto, os resultados de desempenho (Tabela 1) dos animais permitem inferir que ambos os sistemas alimentares são tecnicamente eficientes para a terminação de ovelhas de descarte.

Entretanto, considerando o tempo de permanência dos animais na pastagem e que o tempo médio de utilização de pastagens de aveia-preta + azevém, quando bem manejadas, pode chegar a aproximadamente 120 dias (Rocha et al., 2004), restariam 70 dias para a utilização da pastagem por outras categorias ou mesmo para terminação de outro lote de ovelhas. Há necessidade, contudo, de uma avaliação mais específica da viabilidade econômica desses dois sistemas de alimentação. Furusho-Garcia et al. (2000), avaliando as características da carcaça de animais Texel $\times$ Bergamácia, Texel × Santa Inês e Santa Inês puros alimentados em confinamento com diversas dietas, também verificaram diferenças apenas entre os grupos genéticos, embora os trabalhos tenham sido realizados com cordeiros.

Não foi observada interação raça $\times$ sistema alimentar $(\mathrm{P}>0,05)$ para os pesos absoluto (kg) e relativo (\%) de perna, paleta, costela e pescoço (Tabelas 4 e 5). Os animais da raça Texel foram superiores $(\mathrm{P}<0,05)$ quanto aos pesos de todos os cortes avaliados. Esses resultados estão relacionados ao maior peso médio de carcaça fria desses animais, que foi de 27,33 kg, ou seja, 45,91\% superior ao dos animais da raça Ideal. A proporcionalidade dos cortes em relação à carcaça, no entanto, não diferiu $(\mathrm{P}>0,05)$ entre as raças, resultado que confirma relatos de Oliveira et al. (1998), que, apesar de terem estudado cordeiros, também não notaram diferenças significativas nas proporções de cortes entre as raças Texel e Ideal. Contudo, os valores encontrados neste estudo para os percentuais de perna $(37,5 \%)$ e costela $(34,1 \%)$ diferem dos reportados por esses autores, provavelmente em virtude do padrão de crescimento dos cortes da carcaça.

Silva et al. (2000), avaliando o crescimento dos cortes da carcaça de cordeiros abatidos com diversas idades, observaram redução na proporção de perna e aumento na de costela conforme aumentaram a idade de abate, fato relacionado à maior deposição de gordura na costela com o aumento da idade (Rosa et al., 2002).

O sistema alimentar não afetou $(\mathrm{P}>0,05)$ os pesos e as proporções dos cortes da carcaça, o que evidencia a eficiência de ambos os sistemas de engorda de ovelhas de descarte. Tonetto et al. (2004), avaliando sistemas de terminação de cordeiros, observaram maior proporção de costela nos animais terminados em pastagem cultivada e associaram esse resultado ao maior peso de corpo vazio dos animais em comparação àqueles terminados em confinamento, o que não ocorreu neste estudo.

Não foi observada interação $(\mathrm{P}>0,05)$ raça $\times$ sistema de alimentação para a composição física da perna. O sistema de alimentação não afetou $(\mathrm{P}>0,05)$ essas características, todavia, os pesos de músculos, gordura e ossos foram maiores nos animais da raça Texel, o que está relacionado ao maior peso de perna desses animais, que foi aproximadamente $45,2 \%$ superior ao dos animais da raça Ideal (Tabela 4). 
Tabela 3 - Comprimentos de carcaça (CC) e de perna (CP), largura (LP) e profundidade de perna (PP), profundidade de peito (PPt) e área de olho-de-lombo (AOL) de ovelhas Ideal e Texel terminadas em dois sistemas de alimentação

\begin{tabular}{|c|c|c|c|c|c|c|}
\hline & CC $(\mathrm{cm})$ & $\mathrm{CP}(\mathrm{cm})$ & $\mathrm{LP}(\mathrm{cm})$ & $\mathrm{PP}(\mathrm{cm})$ & PPt $(\mathrm{cm})$ & $\mathrm{AOL}\left(\mathrm{cm}^{2}\right)$ \\
\hline Ideal & 63,2 & 34,6 & 10,4 & 13,9 & 29,3 & 11,9 \\
\hline $\mathrm{P}^{\mathrm{a}}$ & $* * *$ & $*$ & $* * *$ & $* * *$ & $* * *$ & $* * *$ \\
\hline \multicolumn{7}{|l|}{ Sistema alimentar } \\
\hline Confinamento & 66,1 & 35,1 & 11,4 & 15,3 & 29,8 & 13,2 \\
\hline \multicolumn{7}{|c|}{ Raça $\times$ sistema de alimentação } \\
\hline $\mathrm{P}^{\mathrm{a}}$ & ns & ns & ns & ns & ns & ns \\
\hline Média & 66,4 & 35,2 & 11,4 & 15,4 & 30,3 & 14,1 \\
\hline $\mathrm{CV}, \%$ & 2,91 & 3,77 & 7,20 & 7,08 & 4,81 & 14,52 \\
\hline
\end{tabular}

aprobabilidade dos efeitos de raça, sistema alimentar e da interação raça $\times$ sistema alimentar, em que ns $=$ não-significativo, ${ }^{*} P<0,05$ e ${ }^{* * *} P<0,001$; $\mathrm{CV}=$ coeficiente de variação.

Tabela 4 - Pesos absolutos $(\mathrm{kg})$ de perna, paleta, costela e pescoço de ovelhas Ideal e Texel terminadas em dois sistemas de alimentação

\begin{tabular}{|c|c|c|c|c|}
\hline & Perna (kg) & Paleta (kg) & Costela (kg) & Pescoço (kg) \\
\hline \multicolumn{5}{|l|}{ Raça } \\
\hline Ideal & 3,01 & 1,72 & 4,03 & 0,61 \\
\hline $\mathrm{P}^{\mathrm{a}}$ & $* * *$ & $* * *$ & $* * *$ & $* * *$ \\
\hline \multicolumn{5}{|l|}{ Sistema alimentar } \\
\hline $\mathrm{P}^{\mathrm{a}}$ & ns & ns & ns & ns \\
\hline \multicolumn{5}{|c|}{ Raça $\times$ sistema de alimentação } \\
\hline $\mathrm{P}^{\mathrm{a}}$ & ns & ns & ns & ns \\
\hline Média & 3,69 & 2,16 & 4,95 & 0,72 \\
\hline $\mathrm{CV}, \%$ & 9,13 & 8,56 & 14,33 & 16,99 \\
\hline
\end{tabular}

aprobabilidade dos efeitos de raça, sistema alimentar e da interação raça $x$ sistema alimentar, em que ns = não-significativo e ${ }^{\star \star \star} P<0,001 ; C V=$ coeficiente de variação.

Tabela 5 - Pesos relativos (\%) de perna, paleta costela e pescoço de ovelhas Ideal e Texel terminadas em dois sistemas de alimentação

\begin{tabular}{|c|c|c|c|c|}
\hline & Perna $(\%)$ & Paleta (\%) & Costela $(\%)$ & Pescoço (\%) \\
\hline Ideal & 32,2 & 18,4 & 42,8 & 6,5 \\
\hline $\mathrm{P}^{\mathrm{a}}$ & ns & ns & ns & ns \\
\hline \multicolumn{5}{|l|}{ Sistema alimentar } \\
\hline Confinamento & 32,5 & 19,1 & 42,0 & 6,5 \\
\hline \multicolumn{5}{|c|}{ Raça $\times$ sistema de alimentação } \\
\hline $\mathrm{P}^{\mathrm{a}}$ & ns & ns & ns & ns \\
\hline Média & 32,1 & 18,6 & 42,8 & 6,3 \\
\hline CV, \% & 5,03 & 4,44 & 4,55 & 12,07 \\
\hline
\end{tabular}

aprobabilidade dos efeitos de raça, sistema alimentar e da interação raça $x$ sistema alimentar, em que ns = não-significativo $\mathrm{e}^{* * *} \mathrm{P}<0,001 ; \mathrm{CV}=$ coeficiente 
Tabela 6 - Pesos $(\mathrm{kg})$ de músculo, gordura e osso e relação músculo:gordura (RMG) da perna de ovelhas Ideal e Texel terminadas em dois sistemas de alimentação

\begin{tabular}{|c|c|c|c|c|}
\hline & Músculo (kg) & Gordura (kg) & Osso (kg) & RMG \\
\hline \multicolumn{5}{|l|}{ Raça } \\
\hline Ideal & 2,00 & 0,43 & 0,56 & 4,87 \\
\hline Texel & 3,02 & 0,59 & 0,74 & 5,50 \\
\hline $\mathrm{P}^{\mathrm{a}}$ & $* * *$ & $* *$ & $* * *$ & ns \\
\hline \multicolumn{5}{|l|}{ Sistema alimentar } \\
\hline Confinamento & 2,44 & 0,50 & 0,63 & 5,03 \\
\hline Pastagem & 2,58 & 0,53 & 0,67 & 5,33 \\
\hline $\mathrm{P}^{\mathrm{a}}$ & ns & ns & ns & ns \\
\hline \multicolumn{5}{|l|}{$\begin{array}{l}\text { Raça } \times \text { sistema } \\
\text { de alimentação }\end{array}$} \\
\hline $\mathrm{P}^{\mathrm{a}}$ & ns & ns & ns & ns \\
\hline Média & 2,51 & 0,51 & 0,65 & 5,19 \\
\hline $\mathrm{CV}, \%$ & 9,50 & 27,06 & 13,88 & 28,20 \\
\hline
\end{tabular}

aprobabilidade dos efeitos de raça, sistema alimentar e da interação raça $\times$ sistema de alimentação, em que ns $=$ não-significativo, ${ }^{*} \mathrm{P}<0,01 \mathrm{e}$ ${ }^{* * *} \mathrm{P}<0,001 ; \mathrm{CV}=$ coeficiente de variação.

Tabela 7 - Proporções (\%) de gordura e osso da perna de ovelhas Ideal e Texel terminadas em dois sistemas de alimentação

\begin{tabular}{lcc}
\hline & Gordura & Osso \\
\hline Raça & & \\
Ideal & 14,3 & 18,8 \\
Texel & 13,6 & 16,9 \\
$\mathrm{P}^{\mathrm{a}}$ & $\mathrm{ns}$ & $*$ \\
Sistema alimentar & & \\
Confinamento & 14,3 & 17,7 \\
Pastagem & 13,6 & 18,0 \\
$\mathrm{P}^{\mathrm{a}}$ & $\mathrm{ns}$ & $\mathrm{ns}$ \\
Raça $\times$ sistema de alimentação & \multicolumn{2}{c}{} \\
Pa & $\mathrm{ns}$ & $\mathrm{ns}$ \\
Média & 13,9 & 17,8 \\
CV, \% & 21,66 & 9,95 \\
\hline
\end{tabular}

aprobabilidade dos efeitos de raça, sistema alimentar e da interação raça $\times$ sistema alimentar, em que $\mathrm{ns}=$ não-significativo e ${ }^{*} \mathrm{P}<0,05$; $\mathrm{CV}=$ coeficiente de variação.

Tabela 8 - Proporções (\%) de músculo da perna de ovelhas Texel e Ideal terminadas em dois sistemas de alimentação

\begin{tabular}{llccc}
\hline & & \multicolumn{2}{c}{ Raça } & \\
\cline { 3 - 4 } & & Ideal & Texel & CV (\%) \\
\hline \multirow{2}{*}{ Sistema alimentar } & Confinamento & $65,4^{\mathrm{b}}$ & $70,6^{\mathrm{a}}$ & 5,18 \\
& Pastagem & $68,4^{\text {ab }}$ & $68,4^{\text {ab }}$ & 4,01 \\
& CV, \% & 4,24 & 4,13 & - \\
\hline
\end{tabular}

Valores seguidos de letras semelhantes não diferem $(P>0,05)$ entre si pelo teste Tukey.
Quando expresso em valores percentuais (Tabela 7), o peso de ossos foi maior nos animais da raça Texel. O percentual de gordura, no entanto, não diferiu entre os grupos genéticos, o que pode estar relacionado ao fato de os animais terem sido abatidos com mesma condição corporal.

O grupo genético não influenciou $(\mathrm{P}>0,05)$ a relação músculo:gordura (Tabela 6), o que contraria os resultados obtidos por Oliveira et al. (1998), que, em cordeiros, observaram maior relação músculo:gordura nos animais da raça Texel. Neste estudo, observou-se significativa redução na proporção de músculo e acréscimo na proporção de gordura com o avanço da idade, o que está de acordo com relatos de Sainz (1996) de que, com o aumento do peso vivo ou do grau de maturidade, aumenta a proporção de gordura na carcaça dos animais. O sistema alimentar não afetou $(\mathrm{P}>0,05)$ as proporções de gordura e ossos na perna dos animais. Observou-se interação significativa $(P=0,0445)$ raça $\times$ sistema alimentar para a proporção de músculo (Tabela 8), que foi maior nas ovelhas da raça Texel terminadas em confinamento em comparação àquelas terminadas em pastagem cultivada.

\section{Conclusões}

O confinamento e a pastagem cultivada de aveia-preta + azevém são sistemas alimentares tecnicamente equivalentes para terminação de ovelhas de descarte. Ovelhas de descarte da raça Texel, em comparação às da raça Ideal, apresentam maior peso e rendimento de carcaça, além de maiores cortes comerciais.

\section{Literatura Citada}

AGRICULTURAL RESEARCH COUNCIL - ARC. The nutrient requirements of ruminant livestock. London: Commonwealth Agricultural Bureaux, 1980. 351p.

FERREIRA, M.A.; VALADARES FILHO, S.C.; VÉRAS, A.S.C. et al. Predição da composição corporal por intermédio de método indireto. Revista Brasileira de Zootecnia, v.30, n.1, p.242-246, 2001.

FRESCURA, R.B.M.; PIRES, C.C.; ROCHA, M.G. et al. Sistemas de alimentação na produção de cordeiros para abate aos $28 \mathrm{~kg}$. Revista Brasileira de Zootecnia, v.34, n.4, p.1267-1277, 2005.

FURUSHO-GARCIA, I.F.; PEREZ, J.R.O.; OLIVEIRA, M.V. Características de carcaça de cordeiros Texel x Bergamácia, Texel x Santa Inês e Santa Inês puros, terminados em confinamento, com casca de café como parte da dieta. Revista Brasileira de Zootecnia, v.29, n.1, p.253-260, 2000.

GIOTTO, E. Manual Siter 3.1. Santa Maria: Universidade Federal de Santa Maria, 2001. 187p.

HUIDOBRO, F.R.; CAÑEQUE, V. Producción de carne en corderos de raza Manchega. II. Conformación y estado de engarzamiento de la canal y proporción de piezas en distintos tipos comerciales. Investigación Agraria. Producción y Sanidad Animales, v.8, n.3, p.233-243, 1993.

JONES, H.E.; LEWIS, R.M.; YOUNG, M.J. et al. Changes in muscularity with growth and its relationship with other carcass 
traits in three terminal sire breeds of sheep. Animal Science, v.74, n.2, p.265-275, 2002.

MACEDO, F.A.F.; SIQUEIRA, E.R.; MARTINS, E.N. et al. Qualidade de carcaças de cordeiros Corriedale, Bergamácia x Corriedale e Hampshire Down x Corriedale, terminados em pastagem e confinamento. Revista Brasileira de Zootecnia, v.29, n.5, p.1520-1527, 2000.

MORENO, J.A. Clima do Rio Grande do Sul. Porto Alegre: Secretaria da Agricultura, 1961. 41p.

MOTT, G.O.; LUCAS, H.L. The design, conduct and interpretation of grazing trials on cultivated and improved pastures. In: INTERNA-TIONAL GRASSLAND CONGRESS, 6., 1952, Pensylvania. Proceedings... Pensylvania: State College Press, 1952. p.1380-1385.

MÜLLER, L. Normas para avaliação de carcaça e concurso de carcaças de novilhos. Santa Maria: Universidade Federal de Santa Maria, 1980. 31p.

NERES, M.A.; MONTEIRO, A.L.G.; GARCIA, C.A. et al. Forma física da ração e pesos de abate nas características de carcaça de cordeiros em creep feeding. Revista Brasileira de Zootecnia, v.30, n.3, p.948-954, 2001.

NATIONAL RESEARCH COUNCIL - NRC. Nutrient requirements of sheep. 6.ed. Washington, D.C.: National Academy Press, 1985. $112 p$.

OLIVEIRA, N.M.; OSÓRIO, J.C.S.; MONTEIRO, E.M. Produção de carne em ovinos de cinco genótipos. 4. Composição regional e tecidual. Ciência Rural, v.28, n.1, p.125-129, 1998.

OSÓRIO, J.C.S.; OLIVEIRA, N.M.; NUNES, A.P. et al. Produção de carne em ovinos de cinco genótipos. 3. Perdas e morfologia. Ciência Rural, v.26, n.3, p.477-481, 1996.

OSÓRIO, J.C.S.; OSÓRIO, M.T.M.; JARDIM, P.O. et al. Métodos para avaliação da produção de carne ovina: in vivo, na carcaça e na carne. Pelotas: UFPEL, 1998. 98p.
ROCHA, M.G.; MONTAGNER, D.B.; SANTOS, D.T. et al. Parâmetros produtivos de uma pastagem temperada submetida a alternativas de utilização. Revista Brasileira de Zootecnia, v.33, n.6, p.1386-1395, 2004.

ROSA, G.T.; PIRES, C.C.; SILVA, J.H.S. et al. Crescimento de osso, músculo e gordura dos cortes da carcaça de cordeiros e cordeiras em diferentes métodos de alimentação. Revista Brasileira de Zootecnia, v.31, n.6, p.2283-2289, 2002.

RUSSEL, A.J.F.; DONEY, J.M.; GUNN, R.G. Subjective assessment of body fat in live sheep. Journal of Agricultural Science, v.72, p.451-454, 1969.

SAINZ, D.R. Qualidades das carcaças a da carne ovina e caprina. In: REUNIÃO ANUAL DA SOCIEDADE BRASILEIRA DE ZOOTECNiA, 33., 1996, Fortaleza. Anais... Fortaleza: Sociedade Brasileira de Zootecnia, 1996. p.7.

STATISTICAL ANALISYS SYSTEM - SAS. SAS/STAT ${ }^{\circledR} \mathbf{9 . 1}$ User's guide. Cary: SAS Institute, 2004. 5135p.

SILVA, L.F.; PIRES, C.C.; ZEPPENFELD, C.C. et al. Crescimento de regiões da carcaça de cordeiros abatidos com diferentes pesos. Ciência Rural, v.30, n.3, p.481-484, 2000.

SIQUEIRA, E.R. Confinamento de ovinos. In: SIMPÓSIO PAULISTA DE OVINOCULTURA E ENCONTRO INTERNACIONAL DE OVInOCUltURA, 5. 1999, Botucatu. Anais... Botucatu: Universidade Estadual Paulista "Júlio de Mesquita", 1999. p.52-59.

TONETTO, C.J.; PIRES, C.C.; MÜLLER, L. et al. Rendimentos de cortes da carcaça, características da carne e componentes do peso vivo em cordeiros terminados em três sistemas de alimentação. Revista Brasileira de Zootecnia, v.33, n.1, p.234-241, 2004.

WILM, H.G.; COSTELLO, D.F.; KLIPPLE, G.E. Estimating forage yield by the double sampling methods. Journal of American Society of Agronomy, v.36, n.1, p.194-203, 1944. 